\title{
Delayed Serum Calcium Biochemical Response to Successful Parathyroidectomy in Primary Hyperparathyroidism
}

\author{
Yousof Alabdulkarim, Edgard Nassif
}

Notre-Dame Hospital University of Montreal Chum Montreal, QC, Canada

Address for correspondence: Dr. Edgard Nassif, E-mail: edgard.nassif@umontreal.ca

\begin{abstract}
Background: Parathyroidectomy is considered the standard treatment for primary hyperparathyroidism, however, though the onset of biochemical response is variable and is different from one patient to the other.

Aim: To evaluate the onset of systemic response and the biochemical normalization of serum calcium levels to a successful surgery.

Patients and Methods: In a retrospective fashion, we collected clinical data from 303 patients admitted to our hospital between 2005 and 2008, with a diagnosis of primary hyperparathyroidism after sestamibi localization. The pathology reports, parathyroidectomy results, the preoperative and postoperative parathormone (PTH) and serum calcium levels were reviewed. Response of each patient to the surgery was studied and all the data were analyzed to determine how fast the serum calcium levels drop.

Results: The majority of patients $(72.9 \%, 221 / 303)$ showed a decrease in their serum calcium levels to normal values within $48 \mathrm{~h}$. While in 40 patients it took 72 hours and 42 patients $(13.8 \%)$ had a delayed normalization for more than $72 \mathrm{~h}$. The pathology in the $\mathrm{PH}$ group was predominantly of a single adenoma $80.9 \%$ vs. 19.1 with hyperplasia with a $P$ of 0.03 . Preoperative parathyroid hormone PTH elevation was not significantly deferent between the two groups ( $\mathrm{PH}$ and EN) with a mean of $7.9 \pm 5.36$ vs. $7.41 \pm 14.5 \mathrm{pmol} / \mathrm{L}$ respectively with a $P$ of 0.43 .

Conclusion: The majority of patients with primary hyperparathyroidism (PHP) respond to parathyroidectomy in the form of normalization of their serum calcium levels and PTH within $48 \mathrm{~h}$; however, a certain group of patients will need more than 3 days.
\end{abstract}

Keywords: Biochemical response, calcium, endocrine, hyperparathyroidism

DOI: $10.4103 / 0974-2727.66700$

www.jlponline.org

\section{INTRODUCTION}

O arathyroidectomy is a successful and costeffective modality of treatment for primary hyperparathyroidism (PHP) ${ }^{[1-4]}$ In general, the surgery itself is considered to be relatively easy and safe especially in experienced hands. ${ }^{[5,6]}$

The surgery has undergone various modifications in the technique ${ }^{[7]}$ and extent of resection. And whether the surgeon chooses to employ a classical approach, minimal invasive, or video-assisted mini procedure with or without intra-operative radioactive guidance, perform a post resection confirmatory test or not, the ultimate aim is to ensure the successful and complete resection of the hyper-secreting gland and to achieve a full resolution of the biochemical elevation of calcium levels -even if clinically asymptomatic- and eventual returning to a baseline of normal serum calcium levels ${ }^{[3]}$ during follow up. ${ }^{[2]}$

The success of surgery can - reasonably - be confirmed by intra-operative rapid parathormone (PTH) sampling before and immediately after resection of the hyper secreting gland. ${ }^{[8-11]}$ Histopathology resultson the other hand-give a valuable confirmatory data and provide vital information about the type of abnormality (hyperplasia or adenoma) and can detect the relatively rare parathyroid cancer. However, though the crucial determining factor is the clinical resolution of symptomatic disease and the normalization of the serum calcium levels during a sufficient period of follow-up.

Post-operatively, the majority of patients show an early clinical and laboratory response with a marked 
decrease to normal value within the first $24 \mathrm{~h}$, which gives the surgeon an additional reassurance for the success of the intervention while waiting for the definitive histopathology results, However, though it is not unusual to find patients who tend to show a delayed response with persistently elevated calcium for more than $48 \mathrm{~h}$ and in rare occasion this may continue for several days after a successful parathyroidectomy, with eventual improvement later on during the follow up without any further need for reoperation.

It is crucial not to confuse this temporary persistent hypercalcaemia-that is characteristically self-limiting-with the occasional failure of the surgery or the presence of a double adenoma that gives rise to a similar clinical picture marked by the persistent serum calcium and PTH elevation and transient, if any, evidence of response to the first surgery. In the other extreme, a certain group of patients respond with early and prolonged hypocalcaemia.

The understanding of the various behavioral patterns of serum calcium is not only important to reassure both the patient and the treating physician but also to avoid unnecessary prolonged hospital stay and over investigation.

In this study we present the experience of our institute with both challenging situations with more concentration on the first subtype.

\section{PATIENTS AND METHODS}

\section{Patients}

All patients diagnosed with PHP who were referred to the hospital of Notre-dame (CHUM) in Montreal, between January 2005 and July 2008. Three hundred and three patients were considered for inclusion.

Every patient had his biological parameters recorded, parathyroid hormone, and calcium levels preoperative and post-operatively every $12 \mathrm{~h}$.

Only patients who had surgical intervention were included, and as per the hospital protocol they all had a preoperative localization study in the form of Sestamibi ( $99 \mathrm{mTc})$ scan, they were all operated by the same surgeon.

Exclusion criteria were secondary hyperparathyroidism, combined thyroid and parathyroid surgery in one setting, the inability to retrieve the localization study, PTH or calcium level, and any missing follow up records after discharge.

\section{Procedure}

Surgery was performed as a standard anterior approach under general and occasionally local anesthesia (22 patients); partial parathyroidectomy was performed depending on the results of sestamibi scan and intra-operative clinical judgment.

Conformational rapid PTH was requested within $30 \mathrm{~min}$ postoperatively in the recovery room, positive results were considered in case of significant drop of PTH by more than $50 \%$ from that of preoperative.

Calcium levels were estimated as total and ionized serum calcium level in the recovery room and every $12 \mathrm{~h}$, the range of serum calcium was considered normal if between $(1.16-1.26 \mathrm{mmol} / \mathrm{L})$ according to the reference value range in our laboratory.

The histopathology confirmation was not taken as positive till the final report was issued, and was positive for a hypersecretory gland.

\section{Statistics}

Data were collected utilizing Microsoft Excel $^{\circledR} 2003$, they were analyzed using SPSS for windows ${ }^{\circledR}$ 14.0. We expressed our results in Mean \pm SD.

The comparison of results was performed by t-test and chi-square tests, curves were designed based scattered points to compare paired values whenever needed, final results were considered statistically significant when 2 -tailed $P$-value $<0.05$.

The literature review was performed using PubMed and MDConsult with key words including PHP and response to parathyroidectomy for English articles published between 1980 and 2008. Studies for secondary hyperparathyroidism were excluded.

\section{RESULTS}

Between January 2005 and July 2008, three hundred and three consecutive patients were included, their age was ranging between 20 and 84 years with a mean of $56.6 \pm 13.1$ years. The female to male ratio was 3.3 to 1 .

The weight of the gland was ranging between $0.1 \mathrm{~g}$ and $10 \mathrm{~g}$ with a mean of $0.88 \mathrm{~g}$.

Of the 303 patients $230(75.9 \%)$ had a single adenoma according to the final pathology report, compared to 65 
(21.4\%) patients who were found to have hyperplasia. Eight patients $(2.6 \%)$ had equivocal pathology.

Persistent elevation of calcium postoperatively for more than $72 \mathrm{~h}$ was noticed in 42 patients $(13.8 \%)$, who eventually showed normalization of their serum calcium before discharge from the hospital.

The majority of patients 221 patients (72.9\%) showed an evidence of normalization of serum calcium levels in less than $48 \mathrm{~h}$ post-operative period. The rest of the patients 82 patients needed more than $48 \mathrm{~h}$ to show any decrease in their serum calcium levels, but real delay for more than $72 \mathrm{~h}$ was documented in 42 patients (13.8\%), who were considered to have persistent hypercalcaemia (PH) [Figure 1].

\section{Relation to histopathology}

The majority of patients who had persistent hypercalcaemia for more than $72 \mathrm{~h}$ had a pathology suggestive of parathyroid adenoma rather than hyperplasia, 34/42 patients $(80.9 \%$ ) versus $8 / 42$ patients $(19.1 \%)$, respectively, with a $P$ value of 0.03 .

\section{Relation to parathyroid hormones}

The PTH level for each group was recorded and analyzed, and for the group of persistent hypercalcaemia, the mean value of PTH was $7.9 \pm 5.36 \mathrm{pmol} / \mathrm{L}(2.00-20.8 \mathrm{pmol} / \mathrm{L})$; compared to those with early normalization which was in the range of $(0.2-113.8 \mathrm{pmol} / \mathrm{L})$ with a mean of $7.41 \pm 14.5$ $\mathrm{pmol} / \mathrm{L}$ with a $P 0.43$.

\section{DISCUSSION}

The importance of proper control of serum calcium level comes from the fact that there is a clear evidence of higher mortality caused by cardiovascular arres $\mathrm{t}^{[12,13]}$ not to mention the higher incidence of renal stones, osteoporosis, and other symptoms such as constipation, fatigue, and weakness. ${ }^{[14]}$

Even if the hyperparathyroidism is treated the abnormal pathological alteration that has already occurred at the level of the heart, particularly the left ventricle, alteration of conductive function is likely to be associated with early death in this particular group of patients compared to other individuals with normal serum calcium levels. ${ }^{[14-16]}$

Accordingly the general strategy of treatment of symptomatic hypercalcaemia is to establish an early

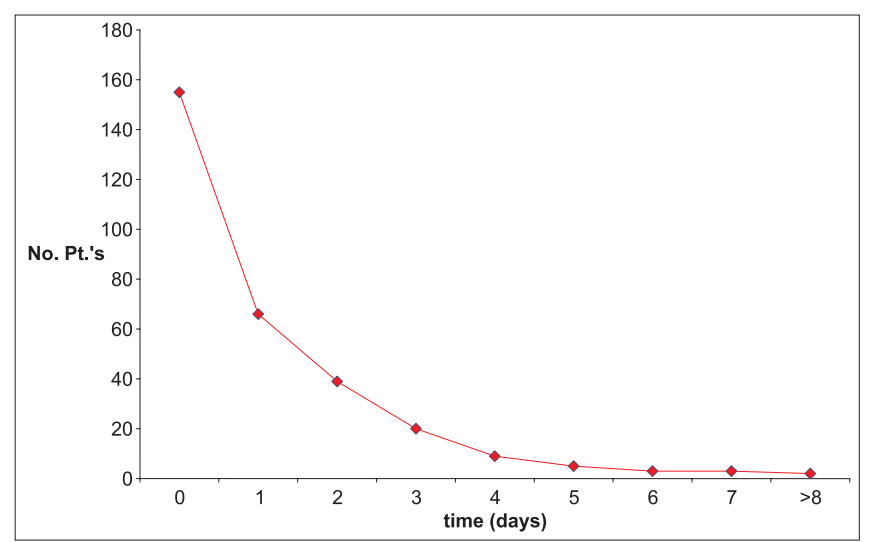

Figure 1: Time (in days) between surgical excisions (horizontal) compared to the number of patients (vertical)

diagnosis followed by appropriate localization study, and invariably the early surgical intervention aiming for cure. ${ }^{[17]}$

The post-operative period is usually of particular importance for the treating team, due to the variable out come of surgery even if this surgery was successful.

For many surgeons who practice in centers with low volume of patients, the failure of calcium levels to drop to normal values is an indicator of either unsuccessful procedure or the presence of double adenoma.

However, though some of the experienced surgeons recognize a subgroup of patients who may show a delayed response with persistently elevated levels of serum calcium that may last for more than 3 days and, in rare occasions, up to 5 days.

This was the case in 42 patients (13.8\%) of our 303 patient populations; however, all of these patients eventually showed gradual but clear and prolonged normalization.

Having the dramatic drop of PTH between the preoperative and post-operative period, the final histopathology reports and the clinical follow up in the OPD as criteria for the success of the surgery indicated a reasonably high levels of curative procedures with only eight patients with equivocal pathology reports.

In general, the time period required for the serum ionized calcium to drop to normal levels-(1.10-1.27 mmol/L) according to our laboratory reference-was between 12 and $48 \mathrm{~h}$, as the records indicate for our population of patients $72.9 \%$.

From pathology point of view, our study reinforced the fact that the adenoma represents the majority of the 
pathological abnormalities causing the elevated secretion of PTH in these PHP patients, and consequently the augmentation of hypercalcaemia, since $75.9 \%$ of the pathology reports showed a single adenoma compared to $21.4 \%$ with an evidence of hyperplasia.

But when considering the type of pathological abnormalities in the subgroup of patients who have a persistent hypercalcaemia there is a clear predominance of adenomas $80.9 \%$ compared to $19.1 \%$ that was associated with a hyperplasia with a $P$ value of 0.03 .

There was no clear relation between the levels of preoperative PTH and the prolonged elevation of calcium despite the predominance of adenomas in the pathology. The mean values of pre-operative PTH were more or less comparable for both groups with a $P$ value of 0.43 ; this might be explained by the small population of the subgroup of patients with this abnormality compared to the rest of the operated patients.

\section{Postulated theory}

The fear of severe post-operative hypocalcaemia encouraged many nephrologists to prescribe oral calcium as prophylactic measures, although it is always discontinued preoperatively by the surgical team, we cannot exclude this as a contributing factor in this phenomena. But in order to clearly understand the mechanism of this type of anomaly in the absence of a double adenoma or failure of surgery, more extensive investigation needs to be done at the ultra microscopic level of calcium metabolism at many levels, e.g. the rate of calcium elimination and bone resorption.

\section{Difficulties}

Some of the patients included in the study were discharged home with calcium supplements and during follow up were discovered to have a transient elevation of their serum calcium level that recovered after cessation of calcium. This need to be followed up for a longer period to assure the accuracy of results.

Many patients had to be excluded due to abnormal renal or hepatic parameters to avoid confusing results which decreased the total sample population.

\section{CONCLUSIONS}

Despite meticulous parathyroidectomy procedures in experienced hands, there will be a subgroup of patients who tend to have a delayed normalization of their calcium levels postoperatively and if the other parameters of successful procedure are met, including intra-operative PTH confirmation, histopathology diagnosis and eventual drop to normal or subnormal levels during follow up, we would like to believe that it might represent a normal variation of response in the post-parathyroidectomy period for aPHP, particularly if caused by a single adenoma that is successfully resected.

\section{REFERENCES}

1. Adamek S, Libansky P, Tvrdon J, Pafko P, Broulík P. Personal experience with a bilateral approach in the surgical treatment of primary hyperparathyroidism: A group of 222 patients with surgery for primary hyperparathyroidism from 1994 to 2000. Rozhl Chir 2002;81:443-9.

2. Allen M, Almeida I, Torrinha J. Surgical treatment of primary hyperparathyroidism with intraoperative iPTH determination. Acta Med Port 2008;21:49-54.

3. Barth A, Dambacher MA, Burgi H, Maurer W. New aspects in the surgical treatment of hyperparathyroidism. Schweiz Med Wochenschr 1987;117:574-8.

4. Dubost C, Charbonnier JY, Assens P. Surgical treatment of primary hyperparathyroidism in patients over 70 years of age. Presse Med 1984;13:1773-5.

5. Brasso K, Karstrup S, Lundby CM, Kristensen LO, Holm HH. Surgical treatment of primary hyperparathyroidism. Dan Med Bull 1994;41:585-8.

6. Brothers TE, Thompson NW. Surgical treatment of primary hyperparathyroidism in elderly patients. Acta Chir Scand 1987;153:175-8.

7. Bonnichon P. Treatment of primary hyperparathyroidism at Cochin hospital (Paris): Changes in procedures during the last thirty years. Ann Endocrinol (Paris) 2008;69:169-73.

8. Bergson EJ, Sznyter LA, Dubner S, Palestro CJ, Heller KS. Sestamibi scans and intraoperative parathyroid hormone measurement in the treatment of primary hyperparathyroidism. Arch Otolaryngol Head Neck Surg 2004;130:87-91.

9. Ferrer Baixauli F, Munoz Barranco A, Alberola Terol V, Espuch Núñez D, Martín del Guayo G, Infante Matarredona E. Surgical treatment of primary hyperparathyroidism: A descriptive analysis. Acta Otorrinolaringol Esp 2004;55:288-94.

10. Ferrer Baixauli F, Severa Ferrandiz G, Menor Ibanez F, Infante Matarredona E. PTHi value in the surgical treatment of hyperparathyroidism. An Otorrinolaringol Ibero Am 2004;31:609-19.

11. Yang ZQ, Zhu LW, Wang PZ. Diagnosis and surgical treatment of 48 cases of parathyroid adenoma and parathyroid carcinoma. Zhonghua Zhong Liu Za Zhi 2006;28:625-7.

12. Barna I, Varadi A, Lakatos P. Metabolic abnormalities related to cardiovascular risk in primary hyperparathyroidism: Effects of surgical treatment. J Intern Med 1999;245:311-3.

13. Hedback G, Oden A, Tisell LE. Parathyroid adenoma weight and the risk of death after treatment for primary hyperparathyroidism. Surgery 1995;117:134-9.

14. Andersson P, Rydberg E, Willenheimer R. Primary hyperparathyroidism and heart disease: A review. Eur Heart J 2004;25:1776-87.

15. Garcia de la Torre N, Wass JA, Turner HE. Parathyroid adenomas and cardiovascular risk. Endocr Relat Cancer 2003;10:309-22.

16. Smith JC, Page MD, John R, Wheeler MH, Cockcroft JR, Scanlon $\mathrm{MF}$, et al. Augmentation of central arterial pressure in mild primary hyperparathyroidism. J Clin Endocrinol Metab 2000;85:3515-9.

17. Nilsson IL, Aberg J, Rastad J, Lind L. Endothelial vasodilatory dysfunction in primary hyperparathyroidism is reversed after parathyroidectomy. Surgery 1999;126:1049-55.

Source of Support: Nil, Conflict of Interest: None declared. 